\title{
Retratamento em laminados cerâmicos minimamente invasivos: Uma revisão integrativa da literatura
}

\author{
Retracting in minimally invasive ceramic laminates: An integrative literature review \\ Retracción en laminados cerámicos mínimamente invasivos: Una revisión integradora de la
}

literatura

Recebido: 19/03/2021 | Revisado: 28/03/2021 | Aceito: 08/04/2021 | Publicado: 17/04/2021

\author{
José Artur Miranda Sousa \\ ORCID: https://orcid.org/0000-0002-5445-7140 \\ Faculdade Santa Maria, Brasil \\ E-mail: artursjp@hotmail.com \\ Karla Beatriz de Freitas Lira \\ ORCID: https://orcid.org/0000-0002-9169-9560 \\ Universidade Estadual de Campinas, Brasil \\ E-mail: karlabeatrizlira@ hotmail.com \\ Daniel Felipe Fernandes Paiva \\ ORCID: https://orcid.org/0000-0003-4186-9856 \\ Universidade Estadual de Campinas, Brasil \\ E-mail: daniel.fernandes.paiva@gmail.com \\ Pedro José Targino Ribeiro \\ ORCID: https://orcid.org/0000-0002-0671-9361 \\ Faculdade Santa Maria, Brasil \\ E-mail: drpedrotargino@gmail.com \\ Frank Gigianne Texeira e Silva \\ ORCID: https://orcid.org/0000-0001-5115-8586 \\ Faculdade Santa Maria, Brasil \\ E-mail: frankodonto@gmail.com \\ Luan Éverton Galdino Barnabé \\ ORCID: https://orcid.org/0000-0001-9657-5180 \\ Faculdade Santa Maria, Brasil \\ E-mail: luanevertongb@ hotmail.com \\ Rodolfo de Abreu Carolino \\ ORCID: https://orcid.org/0000-0002-7962-024X \\ Faculdade Santa Maria, Brasil \\ E-mail: rodolfofoog@yahoo.com.br
}

\begin{abstract}
Resumo
Os laminados cerâmicos minimamente invasivos popularmente conhecidos como "lentes de contato" são finas camadas de porcelana que recobrem a face vestibular dos dentes anteriores com a finalidade de modificar seu tamanho, forma e cor. Elas vêm se popularizando cada vez mais por sua enorme divulgação de resultados belos em diversos meios de comunicação, mas principalmente por ter um protocolo de execução mais conservador, com menos agressão aos tecidos dentários do que as facetas tradicionais. Dessa forma, o objetivo deste trabalho foi avaliar as causas de falhas que culminam no retratamento de restaurações estéticas de laminados cerâmicos minimamente invasivos e como a literatura guia o manjo clínico diante desta situação. Para isso, foi realizada uma revisão integrativa da literatura. Serão selecionados estudos que tenham relevância e sejam pertinentes com o título proposto: Retratamento em laminados cerâmicos minimamente invasivos. Selecionando estudos completos, nos idiomas português e inglês, a depender da plataforma de dados, que tratassem ou estudassem o tema em questão. Incluindo na pesquisa artigos que tratassem deste tema, entretanto não foram selecionados para esta revisão documentos acadêmicos que apresentassem no resumo distanciamento do tema pesquisado ou que apresentassem conflitos de interesse.
\end{abstract}

Palavras-chave: Estética na odontologia; Lentes de contato; Lentes de contato dental.

\section{Abstract}

The minimally invasive ceramic laminates popularly known as "contact lenses" are thin layers of porcelain that cover the buccal surface of the anterior teeth in order to modify their size, shape and color. They are becoming more and more popular due to their enormous dissemination of beautiful results in different media, but mainly because they have a more conservative execution protocol, with less aggression to dental tissues than traditional facets. Thus, the objective of this work was to evaluate the causes of failures that culminate in the retreatment of aesthetic restorations 
of minimally invasive ceramic veneers and how the literature guides the clinical management in this situation. For that, an integrative literature review was carried out. Studies that are relevant and relevant with the proposed title will be selected: Retreatment in minimally invasive ceramic laminates. Selecting complete studies, in Portuguese and English, depending on the data platform, that addressed or studied the topic in question. Including articles that dealt with this topic in the research, however, academic documents were not selected for this review that presented in the summary a distance from the researched topic or that presented conflicts of interest.

Keywords: Aesthetics in dentistry; Contact lenses; Dental contact lenses.

\section{Resumen}

Los laminados cerámicos mínimamente invasivos conocidos popularmente como "lentes de contacto" son finas capas de porcelana que recubren la superficie bucal de los dientes anteriores con el fin de modificar su tamaño, forma y color. Se están volviendo cada vez más populares por su enorme difusión de bellos resultados en diferentes medios, pero principalmente porque tienen un protocolo de ejecución más conservador, con menos agresión a los tejidos dentales que las facetas tradicionales. Así, el objetivo de este trabajo fue evaluar las causas de las fallas que culminan en el retratamiento de restauraciones estéticas de carillas cerámicas mínimamente invasivas y cómo la literatura orienta el manejo clínico en esta situación. Para ello se realizó una revisión integradora de la literatura. Se seleccionarán los estudios que sean relevantes y relevantes con el título propuesto: Retratamiento en laminados cerámicos mínimamente invasivos. Seleccionar estudios completos, en portugués e inglés, según la plataforma de datos, que abordaron o estudiaron el tema en cuestión. Incluyendo artículos que abordaron este tema en la investigación, sin embargo, no se seleccionaron para esta revisión documentos académicos que presentaran en el resumen una distancia del tema investigado o que presentaran conflictos de interés.

Palabras clave: Estética en odontología; Lentes de contacto; Lentes de contacto dentales.

\section{Introdução}

Os laminados cerâmicos minimamente invasivos, conhecidos como lentes de contato, são finas camadas de porcelana que são aplicadas na face vestibular dos dentes a fim de modificar sua forma, tamanho e cor. Esta técnica se tornou popular e vem sendo utilizada para restaurações estéticas devido às suas características como cor, resistência, durabilidade e estabilidade química semelhantes aos tecidos dentários (Rodrigues et al., 2012). As lentes de contato além de garantir um preparo minimamente invasivo, oferecem um tratamento mais conservador quando comparadas com o protocolo de facetas de porcelana tradicionais, que são mais agressivos ao tecido dentário (Aquino et al., 2012; Gonzales et al., 2012).

Para seguir os parâmetros da odontologia restauradora, são preconizados procedimentos mais conservadores possíveis, ou "minimamente invasivos", com o propósito de devolver a função e atender às exigências estéticas dos pacientes (Andrade; Romanini, 2004). O cirurgião dentista deve tentar manter ao máximo o equilíbrio do binômio "saúde-função". Significa que o tratamento deve ser cauteloso, especialmente quando se refere à remoção de estrutura dentária hígida em casos onde a modificação estética está sendo visada (Magne; Belser, 2004). Um dos grandes desafios nessas restaurações foi o desenvolvimento de técnicas onde houvesse uma maior interação entre a superfície do esmalte dental e a restauração indireta, mantendo o fator estético. Diante desse fato, foram desenvolvidas técnicas adesivas que permitem maior preservação da estrutura dental respeitando os requisitos estéticos (Pini et al.,2012).

Algumas das principais causas de retratamento em laminados cerâmicos são: seleção equivocada do caso, não planejamento, fraturas, entre outras. Elas também ocorrem quando não se respeita as limitações do trabalho com facetas, como, por exemplo, pacientes que possuem hábitos parafuncionais, dentes com estrutura coronária reduzida, dentes muito vestibularizados e que apresentam apinhamento ou giroversão. Diante disso, devemos ficar cientes de que para que um tratamento possa ter sucesso devemos focar no bom planejamento e se possível, a união com profissionais de outras especialidades da odontologia: periodontia e ortodontia (Gonzalez et al., 2012).

A literatura tem apresentado escassez de informações para guiar protocolos utilizados em retratamento de laminados cerâmicos minimamente invasivos. Diante da necessidade de realizar essa avaliação e de mais informações adicionais, advogase a realização do presente estudo com o intuito de preencher essa lacuna e contribuir com a prática clínica e o embasamento acadêmico. 
Nestas perspectivas, o objetivo do presente estudo será avaliar tanto as causas de falhas no tratamento em si, quanto o proceder em caso de retratamento, elencando e explicando os pontos na qual o cirurgião dentista deve se ater para minimizar falhas no tratamento e o manejo clinico no retratamento. A pergunta norteadora que guiará a presente Revisão Integrativa da literatura será: $\mathrm{O}$ que causa e como se proceder diante da necessidade retratamento em laminados cerâmicos minimamente invasivos.

\section{Metodologia}

O presente estudo trata-se de uma Revisão da literatura que buscou caracterizar o retratamento ou a necessidade de retratamento para restaurações estéticas minimamente invasivas, conhecidas como lentes de contato dentárias. Considerando a crescente busca na área estética e a inserção do Cirurgião-Dentista neste campo, busca-se um aprofundamento deste tema para gerar informações que possam ser úteis para melhor atuação profissional frente a restauração estética com laminados de porcelana. Optou-se pela síntese qualitativa dos dados dando enfoque a análise dos resultados dos trabalhos selecionados seguindo os parâmetros propostos por Pereira et al (2018) e Ludke e Andre (2013).

Para embasamento e aprofundamento do tema buscou-se o acervo literário disponível on line a fim de selecionar os descritores (palavras-chave) que seriam utilizadas para a busca de um maior acervo referencial científico. Em seguida, serão realizadas pesquisas nas bases de dados Scielo e Pubmed utilizando como descritores: "estética na odontologia", "lentes de contato", "lentes de contato dental", em português; e "esthetics in dentistry", "contact lenses", "dental contact lenses", em inglês.

A pergunta norteadora é: quais as principais falhas que resultam em retratamento de lentes de contato? Como procer em casos de retratamento?

Foram utilizadas as seguintes bases de dados: Scielo, Pubmed e Google acadêmico. O período de abrangência para abordagem dos estudos foi de 2010 a 2020. Dentre os artigos disponíveis para consulta de forma integral, foram selecionados principalmente aqueles que demonstraram uma abordagem relacionada ao estudo envolvendo seres humanos, deixando de lado testes in vitro, e englobando pesquisas científicas, estudos de casos, avaliações clínicas e editorais científicos, além de revisões sistemáticas. Utilizou-se os "filtros" oferecidos pelos mecanismos de buscas das plataformas para que mostrem apenas os artigos publicados em Português/inglês para o Scielo e google acadêmico e em inglês para o pubmed, tendo a origem linguística desta plataforma. Os descritores foram utilizados de forma individual e em conjunto buscando selecionar os artigos pertinentes para realização desta revisão.

Foi realizada a análise descritiva e avaliativa dos dados e principais resultados obtidos nos artigos. Para extração de dados dos artigos selecionados, utilizou-se de um instrumento preconizado e previamente elaborado por Souza, Silva e carvalho (2010), a fim de se assegurar que a totalidade dos dados relevantes e minimizar o risco de erros.

\section{Resultados}

Como previamente citado nos procedimentos metodológicos, fez-se o uso dos descritores que guiassem a busca por estudos ligados à tal área. No Quadro 1 são mostrados os resultados de busca quando utilizados com descritores isolados ou de forma integrada usando o conectivo booleano AND. Vale salientar que partir destes resultados de busca, seguiu-se os critérios de seleção de artigos que fossem ser utilizados para construção textual.

Dentre os resultados da pesquisa, o Quadro 1 revela a quantidade de artigos encontrados em cada base de dados, de acordo com o descritor utilizado e, após ser realizada a metodologia de filtragem de artigo. Vale salientar que após o processo de filtragem e mudando a opção de serem apresentados estudos disponíveis no Google acadêmico, encontrou-se resultados, mas usou-se apenas os dados obtidos no Scielo. 
Um método de Filtragem similar ao descrito acima foi utilizado para a Base de dados Pubmed pesquisado em inglês, tendo em vista a origem da língua mãe desta base de dados, encontrando-se diversos estudos. Vale ressaltar que a busca resultou em muitos estudos relacionados a áreas que divergiam do nosso objeto de estudo.

Quadro 1. Resultados da busca de artigos nas plataformas de dados científicos selecionadas para o estudo, de acordo com a base de dados e disposição do uso dos descritores.

\begin{tabular}{|c|c|}
\hline \multicolumn{2}{|c|}{ PubMED (2013-20) } \\
\hline "Cosmetic" AND "Dentistry" & 8.815 \\
\hline $\begin{array}{c}\text { "Dental" AND "Contact" AND } \\
\text { "Lenses" }\end{array}$ & 19 \\
\hline "Restorative" AND "Denstistry" & 7.816 \\
\hline \multicolumn{2}{|c|}{ Scielo (2013-20) } \\
\hline $\begin{array}{c}\text { "Contact" AND "Dental" AND } \\
\text { "Lenses" }\end{array}$ & 1 \\
\hline $\begin{array}{c}\text { "Retreatment" AND “Contact" AND } \\
\text { "Lenses" }\end{array}$ & Zero \\
\hline $\begin{array}{c}\text { "Restorations" } A N D \text { "Anterior" AND } \\
\text { "Teeth" }\end{array}$ & 10 \\
\hline
\end{tabular}

Fonte: Autores.

Como visto no Quadro 1, os resultados da busca demonstram uma gama de estudo, que ao passarem pelos critérios de filtragem estabelecidos pelo presente estudo, resultou na seleção de 7 (sete) artigos. A caraterização dos estudos pode ser vista no Quadro 2 e a discussão de suas informações seguem abaixo.

Quadro 2. Caracterização Sistematizada dos estudos de acordo com Autor/ano, tipos de estudo, local e principais resultados.

\begin{tabular}{|c|c|c|c|}
\hline Autor/ano & Tipo de Estudo & Local & Principais achados \\
\hline Lima. (2013) & Revisão de Literatura & Brasil (RS) & $\begin{array}{c}\text { Embasou o uso dos laminados na reabilitação } \\
\text { estética do sorriso, destacando as marcas } \\
\text { comerciais que são mais populares em sua } \\
\text { confecção. }\end{array}$ \\
\hline Dias. (2015) & Revisão de & Brasil & $\begin{array}{c}\text { Facetas com mínimo desgaste no preparo, } \\
\text { apresentando as vantagens e desvantagens, } \\
\text { indicações e contra indicações desse tipo de } \\
\text { técnica. }\end{array}$ \\
\hline Peralta. (2016) & Revisão de Literatura & $\begin{array}{c}\text { Brasil } \\
\text { (CE) }\end{array}$ & $\begin{array}{c}\text { Abordaram a longevidade, as principais causas } \\
\text { de falhas das lentes de contato dental. }\end{array}$ \\
\hline Zavanelli. (2017) & Relato de caso & $\begin{array}{c}\text { Brasil } \\
\text { (SP) }\end{array}$ & $\begin{array}{c}\text { Planejamento e execução clínica em seu passo } \\
\text { a passo das lentes de contato cerâmicas, } \\
\text { ressaltando protocolo de confecção dos } \\
\text { preparos, moldagem e cimentação das peças } \\
\text { cerâmicas a base de dissilicatio de lítio. }\end{array}$ \\
\hline
\end{tabular}




\begin{tabular}{|c|c|c|c|}
\hline $\begin{array}{c}\text { Zavanelli et al. } \\
\text { (2017) }\end{array}$ & Relato de caso & $\begin{array}{c}\text { Brasil } \\
\text { (SP) }\end{array}$ & $\begin{array}{c}\text { Protocolo clinico passo a passo para a } \\
\text { reabilitação anterior por meio de laminados e } \\
\text { lentes de contato cerâmicos; o estabelecimento } \\
\text { de um bom planejamento clinico gera sucesso } \\
\text { no tratamento e cumpre as expecatitivas do } \\
\text { paciente. }\end{array}$ \\
\hline Oliveira. (2018) & Revisão de Literatura & Brasil & $\begin{array}{l}\text { Contribuição das lentes de contato para } \\
\text { harmonização do sorriso por se enquadrar nos } \\
\text { critérios considerados de um sorriso belo, } \\
\text { atraente e simétrico, com dentes mais claros e } \\
\text { alinhados no arco. }\end{array}$ \\
\hline Soares. (2019) & Revisão de Literatura & Brasil (RO) & $\begin{array}{c}\text { Indicações e limitações dos laminados } \\
\text { cerâmicos avaliando as evidencias cientificas } \\
\text { para este tratamento restaurador. }\end{array}$ \\
\hline
\end{tabular}

Fonte: Autores.

A amostragem composta por 07 artigos indexados nas bases de dados confiáveis relacionadas à área Saúde seguirá sendo discutida por ordem cronológica de exposição. O Presente estudo preferiu abordar estudos mais recentemente publicados para suprir a necessidade de obter-se informações anualizadas acerca do tema. O registro literário mais antigo datou de 2013 e o mais recente de 2019.

Para Lima (2013), o tratamento com lentes de contato deve ser corretamente planejado e obedecer a indicações especificas para que se obtenha sucesso, sendo mais apropriado para discretas alterações de cor, posicionamento dentário ou fechamento de pequenos diastemas. Já para Dias (2015), as facetas cerâmicas passaram a ser empregadas para suprir uma demanda de procedimentos que fossem mais conservadoras do que as próprias coroas totais, permitindo novas composições estéticas com menor desgaste dentário.

Assim, originou uma nova era, que estimulou a confecção das restaurações totalmente em cerâmicas funcionais, estéticas e duráveis. Quando indicadas corretamente, as cerâmicas são uma alternativa restauradora com excelente capacidade de retratar os intrincados efeitos ópticos dos dentes naturais, da mesma maneira que simular e restaurar a rigidez do dente, estabelecendo as propriedades biomecânicas do mesmo; Dias (2015).

De acordo com o passar dos anos na odontologia foi se tirando a tese de que lentes de contato serviam apenas para tratamentos simples e que não existia muita importância das cerâmicas por ser um material consideravelmente "fraco" para a época, hoje graças as pesquisas dos estudiosos possuímos materiais de ótima qualidade (cerâmicas e adesivos) podendo indicar a maioria dos pacientes podendo modificar a estética do sorriso no paciente sem desgastes radicais nem nos dentes nem nos tecidos vizinhos.

Para Carvalho et al. (2012), o sucesso de uma restauração não está apenas no fator estético sendo que outros requisitos precisam ser atendidos independente do material empregado, dentre eles: a resistência e a longevidade.

As facetas cerâmicas somente podem ser indicadas quando uma ótima adesão é praticável, o que significa que quanto maior quantidade de esmalte, melhor. O preparo do dente deve limitar-se principalmente no interior da camada de esmalte, ou deve exibir uma área de esmalte substancia. Skripnik et al. (2016).

Alguns autores ressaltam algumas situações clínicas que não favorecem o planejamento do tratamento com lentes de contato bucal, e que por isso não são indicados (Cardoso et al., 2011; Decurcio et al, 2015; Francci et al., 2011; Okida et al., 2016), são eles: esmalte superficial insuficiente; dentes com alteração de cor; dentes vestibularizados; dentes apinhados; dentes com elevada carga oclusal; hábitos parafuncionais tal como bruxismo; dentes com extensas restaurações; grande destruição coronária; presença de doença periodontal; pacientes com higiene bucal inadequados. 
O bom senso, aliado a um bom planejamento deve ser utilizado quando for começar um tratamento com os laminados, pois mesmo com os materiais de ótima qualidade já citados, ainda existem diversos tipos de pacientes que não estão aptos a receber o tratamento, a exemplo pacientes que possuem má oclusão e que possuam hábitos parafuncionais.

Soares et al. (2012) relatam as vantagens das facetas cerâmicas quando comparadas as facetas confeccionadas com resina composta, salientando de que a estabilidade da cor tem um período de tempo maior, alta resistência de mecânica à fratura, proporcionam a sua longevidade clínica.

No entanto para Costa (2015), mesmo que as cerâmicas apresentem melhores propriedades físicas e mecânicas aos seus antecessores, as suas peculiaridades estruturais dependem não só da microestrutura do material, mas também da sequência dos procedimentos clínicos e laboratoriais durante a confecção e a entrega da restauração, sendo capaz de influenciar na sua durabilidade, mencionando ainda de que a manutenção é um motivo muito importante que contribui a longevidade das lentes de contato dental, pois previne efeitos adversos na gengiva e no dente.

Vantagens e desvantagens das peças de porcelana em relação as resinas compostas quanto sua forma, estabilidade de cor, técnica e preço de execução. Uma ótima alternativa se não fosse o alto valor cobrado para se executar a mesma, temos que salientar também as técnicas de elevado conhecimento e sequencia clinica correta. Já nas resinas o preço e mais acessível, mas não possui a mesma beleza, nem mesmo a durabilidade das lentes de contato.

\section{Discussões}

\subsection{Estética na odontologia}

As expressões faciais, tais como o sorriso, são tão essenciais para a interação pessoais que a literatura científica define o sorriso como a expressão facial mais fortemente associada à manifestação de afeto positivo e de emoções como prazer e alegria. Um sorriso esteticamente agradável (dentes brancos dispostos harmonicamente) gera uma percepção positiva e possibilita ao indivíduo que o vir, satisfação no outro e ao que o detém, uma melhor aceitação e êxito em seu grupo social. (Mesquita \& Mes, 2011).

A atração facial representa uma das mais importantes dimensões da aparência física, procedimentos estéticos na odontologia acrescem a finalidade de devolver a forma e função das estruturas bucais, o objetivo de, na harmonização do sorriso, reforçar o conforto emocional do indivíduo. (Alves, Rezende \& Farjado, 2016).

Com essa busca das pessoas pelo "sorriso perfeito" a odontologia estética encontra-se em um contínuo avanço e tem sido cada vez mais praticada nos últimos anos, em virtude dos procedimentos adesivos e do desenvolvimento de materiais restauradores que buscam a reprodução das características naturais das estruturas dentais. Atualmente, o cirurgião dentista se dispõe de diversas opções de materiais restauradores. Procedimentos diretos e indiretos, com resinas compostas ou cerâmicas, são variáveis que muitas vezes dificultam o correto diagnóstico de qual técnica ou de qual material são mais adequados para cada situação clínica. (Higashi et al., 2012).

O conceito da Odontologia Restauradora atual preconiza que, para qualquer tipo de procedimento, o profissional deve sempre optar pelo tratamento mais conservador, isto é, com maior preservação de estrutura dental sadia. O plano de tratamento deve ser realizado de modo que permita formular um bom prognóstico a médio e longo prazo, não apenas em termos de estética, como também considerando os aspectos biológicos e funcionais. Nenhum tipo de tratamento poderá ter êxito sem o estabelecimento de um correto diagnóstico e adequado planejamento. Esta etapa é, provavelmente, uma das mais importantes e imprescindíveis para a obtenção de excelência (Baratieri, 2001).

\subsection{Lentes de contato dentárias (restaurações de porcelana minimamente invasivas)}

A técnica de reabilitação estética com laminados cerâmicos ultrafinos é relativamente nova, e em ascensão na Odontologia, portanto, apresenta limitados números de estudos e resultados em longo prazo (cerca de 20 anos). Também 
denominados de restaurações cerâmicas minimamente invasivas, trata-se de laminados extremamente conservadores na reabilitação estética do sorriso que são uma ótima opção estética para reabilitação de dentes anteriores devido suas excelentes propriedades já mencionadas. (Oliveira, 2007).

O conceito de laminados cerâmicos surgiu em 1928 quando o dentista californiano Charles Pincus inventou essa técnica para que pudesse ser utilizada durante filmagens, mas caiu no esquecimento por falta de adesividade das peças. Posteriormente, com o advento do sistema adesivo e condicionamento ácido por buonocore em 1955, e a adição da técnica do condicionamento do ácido hidrofluorídrico e silanização, por Simonsen e Calamia em 1982, a reabilitação definitiva através de laminados cerâmicos tornou-se possível pela adesão do cimento resinoso à superfície do dente e à porcelana. (Lima, 2013).

O uso da porcelana na odontologia iniciou-se em 1838, e desde essa época até os dias atuais, constitui-se como o material mais próximo das características de um dente natural. Exigindo um desgaste dental menos acentuado, em diversos casos, e sendo menos invasivo que um preparo para coroa total metalo-cerâmica, os laminados cerâmicos tornaram-se bastante atrativos. Apresentam inúmeras vantagens, pois reúnem as qualidades dos compósitos como a capacidade de adesão ao substrato dental e das cerâmicas, como a estabilidade de cor, alta resistência, coeficiente de expansão térmica semelhante ao do esmalte dental e grande reprodutibilidade estética. (Gilson et al., 2007).

A utilização dessas facetas como alternativa estética se popularizou na década de 80, com o desenvolvimento de peças de cerâmicas prensadas reforçadas por leucita, e o avanço na cimentação adesiva, tornou viável obter peças cada vez menos espessas e com melhor resistência biomecânica (Guess et al., 2011). Atualmente, as facetas utilizadas são extremamente finas, cerca de 0,3 a 0,5 mm, chamadas de lentes de contato dentais devido sua semelhança de espessura e translucidez com as lentes de contato oculares. (Francci et al., 2011). Elas podem ser confeccionadas de maneira tradicional, ou usando a tecnologia CAD/CAM para maior precisão no ajuste.

O sistema CAD/CAM (Computer Aided Design / Computer Aided Manufactured) consiste em um escâner que lê e converte a geometria existente em dados lógicos pelo computador, trata-se de um software de construção e planejamento, e uma tecnologia de produção para gerar o objeto desejado. O aparelho de digitação e uma câmera intraoral que pode dispensar os procedimentos convencionais de moldagem agilizando o tempo clinico. $\mathrm{O}$ sistema de automatização CEREC, o mais antigo sistema CAD/CAM, já é utilizado desde os anos 80 para a confecção de inlays cerâmicos, tendo alta taxa de sucesso clinico e exatidão tridimensional. (Beuer et al., 2008).

A popularização dessa técnica deve-se ao fato de que tanto as pessoas, quanto os cirurgiões-dentistas terem informações sobre lentes de contato através da internet, cursos e outros meios de comunicação. Além disso, e uma técnica que contagia todos os pacientes pela sua beleza, resultado rápido, dispensar o uso de anestesia e o principal, preservar a estrutura dentária. (Lima, 2013).

Com a popularização, também surgiu a demanda por tratamentos de dentes anteriores com laminados cerâmicos, pois apesar das resinas compostas oferecerem excelentes resultados com custos mais acessíveis, elas não tem tanta resistência a mudança de cor e desgaste quanto as cerâmicas atuais que são cimentadas em um preparo mínimo (muito menos desgaste que nas de resina composta) e servem para concertar a maioria dos dentes que possuem alterações de forma, tamanho cor ou posição na cavidade bucal do paciente. (Rucker et al., 1990).

Entretanto, desde que surgiram no mercado, as lentes de contato são questionadas e investigadas pelos estudiosos. A cada novo estudo são descobertas mais técnicas inovadoras sempre visando aumentar a resistência das restaurações, visando um maior tempo de durabilidade na boca do paciente sem precisar de manutenções. A durabilidade das restaurações depende principalmente de: um preparo bem executado, cimentação adequada, orientação ao paciente sobre os cuidados com a mesma e o cuidado do profissional para com a manutenção. (Chalegre, \& Barbosa, 2017). 


\subsection{Retratamento em lentes de contato}

Como já foi citada, a literatura é carente de informações cientificas que foquem realmente no retratamento de laminados cerâmicos, por ser uma técnica "muito nova" há poucos protocolos baseados na evidência científica, portanto, neste ponto iremos tratar basicamente de falhas e da longevidade das facetas, tendo em vista que as mesmas estão ligadas a necessidade de retratamento.

As lentes de contato são um tratamento de grande durabilidade e segurança, porém não são todos os pacientes que realizam o tratamento devido suas situações clinicas, sendo assim, um dos seus pontos negativos. Para ser executado o procedimento deve ser feita uma análise criteriosa de aspectos clínicos como: a cor do substrato dental, a necessidade de preparo, o tipo de cerâmica que vai ser implementada em determinado tipo de paciente e o tipo de cimento resinoso. (Skripnik, 2016).

Os pacientes que apresentam hábitos para funcionais como bruxismo, não são indicados para o tratamento com lentes de contato, mas, é bom frisar que se feito um diagnóstico prévio e alertar o paciente quanto a longevidade clínica do tratamento e confecção de uma placa articular, o mesmo pode ser indicado. (Conceição, 2007).

A literatura demonstra que para se conseguir uma boa eficácia, e consequentemente maior longevidade do tratamento, o ideal é que os laminados estejam em contato com a maior área possível do esmalte. (Aquino et al., 2009). Outra contra indicação são casos em que se observam grandes alterações de cor dos dentes, pois a mesma está baseada na mínima espessura de material utilizado nas lentes, o que as torna translúcidas, dificultando que a técnica possa mascarar a coloração inadequada do dente. (Stoll \& Lopes, 2009)

A indicação correta para as "lentes de contato dentais" seria o ajuste vestibular ou incisal. O problema comum que podemos detectar nas restaurações atuais feitas com esse material é a perda da harmonia anatômica, pois a má aplicação pode gerar a impressão de aparência volumosa, desarmônica e errônea, geralmente quando se tenta reproduzir a anatomia interna. (Kacker et al., 2011).

No critério "falhas", as facetas ultrafinas quando combinadas com cobertura interna inadequada resultam em um aumento na tensão, tanto na superfície quanto na interface dente-restauração. Desta forma, foi constatado que a espessura do material para cimentação tem grande influência quando se leva em consideração a distribuição das tensões nas restaurações. Com o isso, o método convencional de cimentação (cimento adesivo aplicado e polimerizado com o laminado na posição desejada) esteve associado a falhas de adesão entre a camada híbrida e a superposta. Sabendo disso, sugere-se um método alternativo, no qual o cimento é aplicado à dentina e polimerizado antes da cimentação. (Magne et al., 2008).

\section{Conclusão}

Baseado na revisão de literatura exposta, foi possível concluir que os laminados cerâmicos minimamente invasivos é um procedimento que vem conquistando um número muito alto de pacientes, tanto por sua beleza como por sua rapidez e mínima agressão ao tecido dentário.

Não obstante, como foi citado em questão por ser uma técnica muito nova (cerca de 20 anos) há poucos protocolos evidenciados na literatura que realmente foquem no retratamento dos laminados e a carência de artigos dificultam o estudo em questão. Diante disso, focamos na importância do sorriso e das expressões faciais e principalmente em elencar as falhas que ocorrem durante o tratamento tendo vista que as mesmas possam resultar em retratamento e também nos protocolos a serem seguidos para melhor execução das lentes.

Com isso, é importante ressaltar a necessidade de que mais estudos sejam desenvolvidos acerca do assunto, focando principalmente em protocolos de realização de retratamentos dos laminados cerâmicos, visto que os estudos servirão de apoio científico para os profissionais da odontologia, buscando sempre estabelecer o melhor plano de tratamento para os pacientes no 
cotidiano das clínicas odontológicas.

\section{Referências}

Alves-Rezende, M. C. R., Lopes-Mrane, G., \& Zavanelli, A. C. (2015). Acolhimento e bem estar no atendimento odontológico humanizado: o papel da empatia. Arch Health Invest, 4 (3), 57-61.

Andrade, O. S. et al. (2012). Ultimate ceramic veneers: a laboratory-guided ultraconservative preparation concept for maximim enamel preservation. Quintessence Dent. Technol., 35, 29-42.

Aquino, A. P. T. et al. (2009). Porcelain laminate veneers: esthetic and functional solution. Clin Int J Braz Dent, $5,42-152$.

Baratieri, L. N. et al. (2001). Odontologia restauradora: fundamentos e possibilidades. Liv.

Beuer, F. et al. (2008). Fabricação automatizada de restaurações dentarias. In: Baratieri, L.N. et al. Soluções clinicas: fundamentos e técnicas. Florianópolis: Ed. Ponto.

Chalegre, A. \& Barbosa, T., 2017. Longevidade e Resistência dos Laminados Cerâmicos (Lentes de Contato Dentária) Em Reabilitações Estéticas: Uma Revisão de Literatura. Graduação. Faculdade Integrada de Pernambuco.

Conceição, E. N. (2007). Dentística: saúde e estética. Artmed.

Francci, C. et al. (2011). Odontologia estética: soluções minimamente invasivas com cerâmicas. Rev. Fundecto, 10, 8-9.

Gilson, J. G. R. et al. (2007). Restauração Indireta do tipo onlay em empress 2 - Relato de caso clínico. International Journal Of Dentsitry, 6 (2), 67-70.

Gonzalez, M. R. et al. (2011). Falhas em restaurações com facetas laminadas: uma revisão de literatura de 20 anos. Rev. bras. odontol, 68 (2), $238-243$.

Guess, P. C. et al. (2011). All-ceramic systems: laboratory and clinical performance. Dent. Clin. N. Am., 55 (2), $333-352$.

Higashi, C. et al. (2006). Planejamento Estético em Dentes Anteriores. In: MIYASHITA, E. Odontologia Estética: Planejamento e técnica. São Paulo: Editora Artes Médicas Brasil.

Ludke, M. \& Andre, M. E. D. A. (2013). Pesquisas em educação: uma abordagem qualitativa. E.P.U.

Kacker, M. D., Yarovesky, U., \& Jadali, L. (2011). Ultra-thin veneers: beautiful and natural. Dentistry Today, 30 (7), $102-104$.

Lima P., 2013. Laminados cerâmicos minimamente invasivos: uma revisão sobre lentes de contato dentais. Graduação. Universidade Federal do Rio Grande do Sul.

Magne, P. et al. (2013). The case for moderate guided prep. Indirect porcelain veneers in the anterior dentition. The pendulum of porcelain veneer preparations: from almost no-prep to over-prep to no-prep. Eur. J. Esthet. Dent., 8 (3), 376-388.

Mesquita, M., 2011. O sorriso humano. Universidade de Lisboa.

Pereira A. S. et al. (2018). Metodologia da pesquisa científica. UFSM. https://repositorio.ufsm.br/bitstream/handle/1/15824/Lic_Computacao_MetodologiaPesquisa-Cientifica.pdf?sequence $=1$.

Oliveira, M. L. M. et al. (2007). Manutenção periódica preventiva em dentistica. In: Conceição, E. N. Dentistica: Saúde e estética. Artmed.

Pini, N. P. et al. (2012). Advances in dental veneers: materials, applications, andtechniques. Dovepress Journal: Clinical, Cosmetic and Investigational Dentistry, 4 (3), 9-16.

Rodrigues, R. B. et al. (2012). Clareamento dentário associado à facetas Indiretas em cerâmica: Abordagem minimamente invasiva. Rev Odontol Bras Central, $21(59), 520-525$

Rucker, L. M. et al. (1990). Porcelain and resin veneers clinically evaluated: 2-year results. The Journal of the American Dental Association, 121 (5), 594-596.

Skripnik, N. N., 2016. Cerâmicas para facetas em dentes anteriores: uma revisão de literatura. Graduação. Universidade Federal de Santa Catarina.

Stoll, L. B, \& Lopes, F. (2009). Harmonização do sorriso através de laminado cerâmico "lente de contato". Rev. dental press estét., 6 (1), $116-124$. 